American J. of Engineering and Applied Sciences 5 (1): 89-92, 2012

ISSN 1941-7020

(C) 2014 M. Hassan et al., This open access article is distributed under a Creative Commons Attribution

(CC-BY) 3.0 license

\title{
Voice-Based Control of a DC Servo Motor
}

\author{
${ }^{1}$ Musaab Hassan, ${ }^{2}$ Hammam Mahjoub and ${ }^{2}$ Mohammed Obed \\ ${ }^{1}$ Department of Mechanical Engineering, Faculty of Engineering, \\ Sudan University of Sciences and Technology, Southern Campus, Khartoum, Sudan \\ ${ }^{2}$ Department of Electrical Engineering, \\ Faculty of Engineering, University of Khartoum, Khartoum, Sudan
}

\begin{abstract}
Problem statement: Motors play a very important role in our life and among which is the DC servo motors. The techniques of controlling these DC motors are plenty, among which is sound. In this study, a voce-based technique was implemented to control the speed and the direction of rotation for a DC motor. Approach: A Microcontroller-based electronic control circuit was designed and implemented to achieve this goal. Results: The speed of the motor was controlled, in both directions, using pulse width modulation and a microcontroller was used to generate the right signal to be applied to the motor. Conclusion: The loudness of human voice was successfully divided into different levels where each level drives the motor at different speed.
\end{abstract}

Key words: Width modulation, phonetic parameters, Pulse Width Modulation (PWM)

\section{INTRODUCTION}

DC servo motors plays an important role in many engineering applications. Engineers always search for an efficient and easy way to control these motors. This is normally carried out by varying the amount as well as the polarity of the applied voltage (Silva, 2005). Pulse width modulation is often used to control DC motors (Bishop, 2002). Another interesting alternative to control motor is through voice. Voice-operated control have been applied to different applications from medical field like surgical devices (Reichenspurner et al., 1999) to pc functions such as cursor movement (Harada et al., 2008), keyboard (Manaris et al., 2001) and in home applications through sound activated switches and painting (Harada et al., 2007). Some attempts was made to tackle issues related use of voice-control such as; continuous control (Igarashi and Hughes, 2001) traing efforts for voice recognition (Smith et al., 2010), noisy environment (Wijekoon et al., 2004). Acoustic phonetic parameters like pitch, loudness (House et al., 2009), vowel quality can be used to control the motion (Bilmes et al., 2005). Igarashi and Hughes (2001) used the presence of voice to switch on the electronic circuit. (Olwal and Feiner, 2005) considered the speed with which the sentence was spoken. In this study, authors attemps to use voice loudness' parameter to control both the speed as well as the direction of a DC servo motor.

\section{MATERIALS AND METHODS}

As illustrated in Fig. 1, the hardware design is classified into six main stages. Firstly, sound signal is to be changed to an electrical signal. SM58 SHURE dynamic microphone is used here to produce an electrical signal from the sound signal vibration. The electrical output signal from this microphone is to be amplified using a sound amplifier with a high slew rate. LM386 (Low Voltage Audio Power Amplifier) was used here to give the required amplification. The amplification circuit is shown in Fig. 2. The input was applied to the LM386 through $10 \mathrm{~K} \Omega$ variable resistor so as to meet the operating point of the internal transistors. Pin. 1 was connected to pin. 8 through $10 \mu \mathrm{F}$ capacitor to increase the gain to the maximum .pin.7 was connected to the ground through 220 $\mu \mathrm{F}$ as a bypass capacitor to bypass unwanted signals to the ground. After amplification the signal was converted to DC using four diodes as a full wave rectifier as shown in Fig. 3. In order to overcome the problem of rippling, a parallel combination of a capacitor and a resistor was used. The microcontroller (ATMEGA16) has three functions as shown in Fig. 4. The Pulse Width Modulation (PWM) that controls the rotational speed of the motor will be generated from the microcontroller.

The motor driving circuit consists of two parts; the ULN 2003 to protect the microcontroller from high voltage and current and the H-bridge (with 4 MOSFETs and pull up resistors) to control the direction of motor rotation. The complete circuit layout of all the previously mentioned stages is presented in Fig. 5.

Corresponding Author: Musaab Hassan, Department of Mechanical Engineering, Faculty of Engineering, Sudan University of Sciences and Technology, Southern Campus, Khartoum, Sudan 


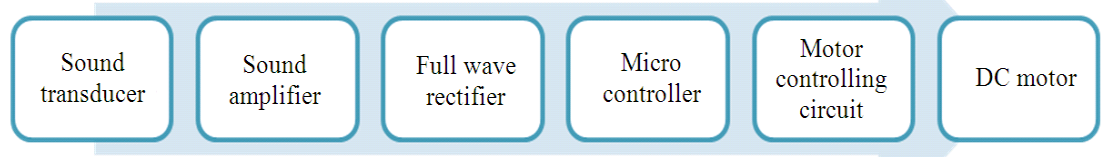

Fig. 1: The main stages of the control system

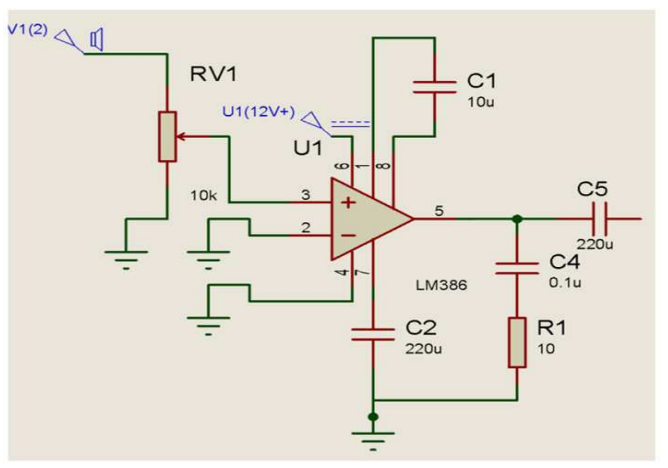

Fig. 2: sound amplifier circuit

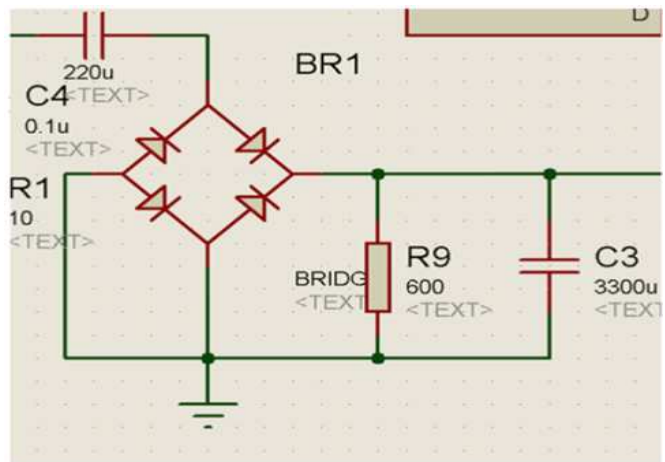

Fig. 3: Full wave rectifier circuit

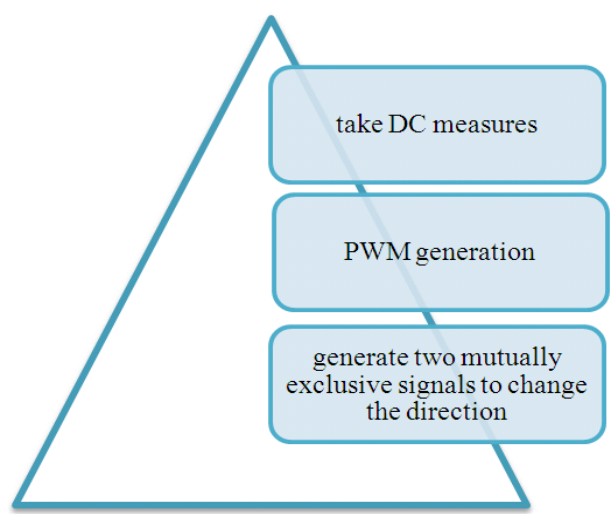

Fig. 4: Microcontroller functions

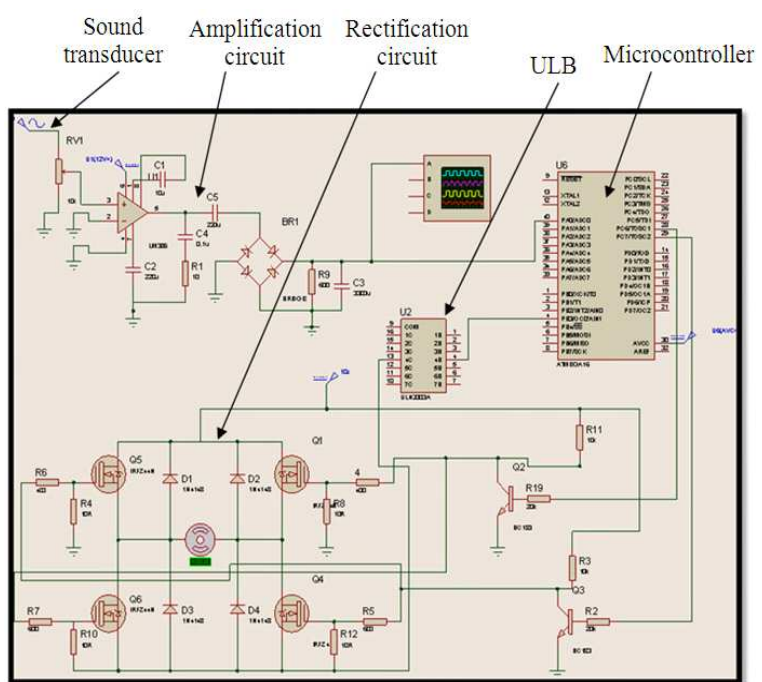

Fig. 5: layout of the control circuit

The final stage of the design was to program the microcontroller to control the speed of the motor. This was achieved by assigning four different speeds (different PWM signals) to four different levels of sound signal. The direction of rotation was set to change when the input voltage, or the sound level, is high (greater than 3.5 volts).

\section{RESULTS}

Prior to the hardware implementation, the speed controlling circuit was simulated in the Proteus simulator and the motor speed was displayed on a screen in the simulation. The level of motor speed was found to vary with the sound level.

As mentioned earlier, the loudness of the sound and its corresponding voltage was divided into four levels as shown in Table 1. Depending on the four levels of sound, the designed circuit should provide different PWM ratios. A comparison between the expected duty cycles and the measured ones is plotted in Fig. 6 while Fig. 7 shows the actual measured cycle as seen on the oscilloscope as compared to expected duty cycle. 


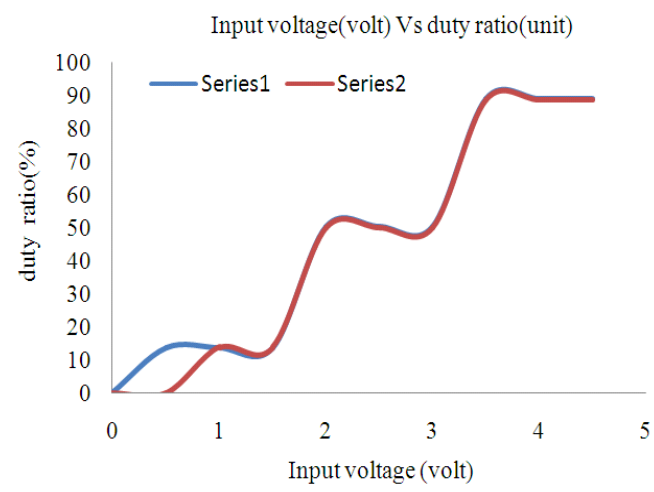

Fig. 6: Generated Vs expected duty cycles at different input voltages
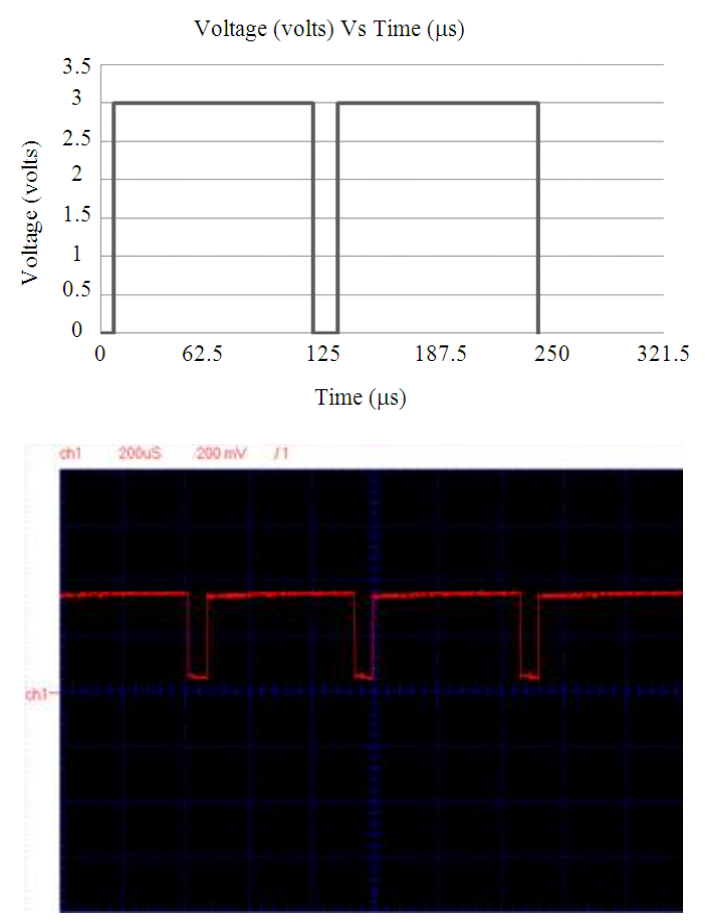

Fig. 7: Expected PWM (left) and generated PWM (right) at high sound level ( $\mathrm{V}=3.8$ volts)

Table 1: Comparison between measures and expected duty cycles

\begin{tabular}{llcl}
\hline Sound level & Input (volts) & $\begin{array}{l}\text { Expected duty } \\
\text { cycle }(\%)\end{array}$ & $\begin{array}{l}\text { Measures duty } \\
\text { cycle }(\%)\end{array}$ \\
\hline Very low & 0.0 & 0.0 & 0.0 \\
Low & 0.5 & 13.7 & 0.0 \\
& 1.0 & 13.7 & 13.8 \\
Medium & 1.5 & 13.7 & 13.8 \\
& 2.0 & 50.2 & 50.0 \\
High & 2.5 & 50.2 & 50.0 \\
& 3.0 & 50.2 & 5.0 \\
& 3.5 & 89.0 & 88.5 \\
& 4.0 & 89.0 & 88.5 \\
\hline
\end{tabular}

\section{DISCUSSION}

A good agreement was found between the expected and measured results as can be seen from Table 1 and Fig. 6. The only error appeared in the results is when the input was 0.5 volt. In this case, the expected duty ratio is $13.7 \%$ but no PWM was detected. This is due to the fact that supplied voltage is slightly less than 0.5 volts and the microcontroller were programmed to consider any value less than 0.5 as nil. It was also observed that the DC motor changes direction of rotation when the amplitude of sound reaches a value between (3.5- 4 volt).

\section{CONCLUSION}

Human voice was used successfully to control the speed, as well as the direction of rotation, of a DC motor. This was accomplished by designing and implanting a microcontroller-based electronic control circuit. Initially, the sound signal was converted to an electrical signal, amplified, rectified and finally applied to a DC motor via a microcontroller. The loudness of the sound was divided into four levels (very low, low, medium and high) and the motor was driven, in both directions, at three different speeds (low, medium and high) using PWM.

\section{REFERECE}

Silva, D.C.W., 2005. Mechatronics: An Integrated Approach. 1st Edn., CRC Press, ISBN-10: 0849312744 pp: 1312.

Bishop, R.H., 2002. The Mechatronics Handbook. 1st Edn., CRC Press, Boca Raton, ISBN-10: 0849300665 pp: 1272.

Reichenspurner, H., R.J. Damiano, M. Mack, D.H. Boehm and H. Gulbins et al., 1999. Use of the voice-controlled and computer-assisted surgical system zeus for endoscopic coronary artery bypass grafting. J. Thorac. Cardiovasc Surg., 118: 11-16. PMID: 10384178

Harada, S. J.A. Malkin, X. Li and J.A. Bilmes, 2008. The vocal joystick: Evaluation of voice-based cursor control techniques for assistive technology. Disabil. Rehab.: Assistive Technol., 3: 22-34. PMID: 18416516

Harada, S., J.O. Wobbrock and J.A. Landay, 2007. Voicedraw: A hands-free voice-driven drawing application for people with motor impairments. The Pennsylvania State University.

Manaris, B., R. McCauley and V. MacGyvers, 2001. An intelligent interface for keyboard and mouse control -- providing full access to pc functionality via speech. Proceedings of the 14th International Florida Artificial Intelligence Research Society Conference, (IFAIRSC' 01), ACM, pp: 182-188. 
Am. J. Engg. \& Applied Sci., 5 (1): 89-92, 2012

Igarashi, T. and J.F. Hughes, 2001. Voice as sound: using non-verbal voice input for interactive control. Proc. UIST, 0: 155-156.

Smith, C.V., J.C. Licato, M.V. Doran and T.G. Thomas $\mathrm{Jr}, 2010$. A voice operated tour planning system for autonomous mobile robots. J. Sys. Cybernetics Inform., 8: 72-79.

Wijekoon, M.P.A., W.T.P. Wjesinghe, G.A.A. Upendra, U.B. Vitharana and T. Nanayakkara, 2004. Sound source localization assisted active security camera. Department of Electrical Engineering, University of Moratuwa.

House, B., J. Malkin and J.A. Bilmes, 2009. The VoiceBot: A voice controlled robot arm. Proceedings of CHI 2008: ACM Conference on Human Factors in Computing Systems, (HFCS' 09), ACM, Boston, MA., pp: 1-10.
Bilmes, J.A., X. Li, J. Malkin, K. Kilanski and R. Wright, 2005. The vocal joystick: A voice-based human-computer interface for individuals with motor impairments. University of Washington.

Olwal, A. and S. Feiner, 2005. Using prosodic features of speech and audio localization in graphical user interfaces. Proceedings of IUI 2005 International Conference on Intelligent User Interfaces, Jan 9-12, San Diego, CA, pp: 284-286. 